\title{
Novas associações de Tephritidae e Lonchaeidae (Diptera) e suas plantas hospedeiras na Amazônia Oriental
}

\author{
Alison Pureza Castilho ${ }^{1}$ \\ Leandro Carvalho da Silva ${ }^{2}$ \\ Maria do Socorro Miranda de Sousa ${ }^{3}$ \\ Jhulie Emille Veloso dos Santos ${ }^{4}$ \\ Walkymário de Paulo Lemos 5 \\ Ricardo Adaime 1,3,6* \\ ${ }^{1}$ Universidade Federal do Amapá, Programa de Pós-graduação da Rede Bionorte \\ CEP 68902-280, Macapá - AP, Brasil \\ ${ }^{2}$ Universidade Federal Rural da Amazônia, Programa de Pós-graduação em Agronomia \\ CEP 66095-780, Belém - PA, Brasil \\ ${ }^{3}$ Universidade Federal do Amapá, Programa de Pós-graduação em Biodiversidade Tropical \\ Macapá - AP, Brasil \\ ${ }^{4}$ Faculdade de Macapá \\ CEP, 68906-801, Macapá - AP, Brasil \\ ${ }^{5}$ Embrapa Amazônia Oriental, Belém \\ Belém - PA, Brasil \\ ${ }^{6}$ Embrapa Amapá, Macapá, Amapá \\ CEP 68903-419, Macapá - AP, Brasil \\ * Autor para correspondência \\ ricardo.adaime@embrapa.br
}

Submetido em 16/04/2019

Aceito para publicação em 09/07/2019

\section{Resumo}

Larvas de Tephritidae e Lonchaeidae (Diptera) infestam frutos de diversas espécies vegetais. O objetivo deste trabalho foi identificar as espécies dessas famílias, suas plantas hospedeiras e parasitoides, em um pomar misto em Belém, Pará, Amazônia Oriental. Foram realizadas amostragens de frutos de diversas espécies vegetais, a cada 30 dias, de novembro de 2017 a maio de 2018. Foram coletadas 109 amostras de frutos (2.355 frutos, $51,7 \mathrm{~kg}$ ), pertencentes a 21 espécies vegetais (oito nativas e 13 introduzidas) de nove famílias botânicas. Houve infestação por larvas de dípteros em 63 amostras (11 espécies de seis famílias botânicas). Foram obtidos espécimes de seis espécies de Tephritidae, três de Lonchaeidae e cinco de parasitoides Braconidae. O maior índice de infestação foi registrado em Spondias mombin, que também apresentou o maior índice de parasitismo. Neste trabalho são registradas novas associações de espécies de Tephritidae e Lonchaeidae e plantas hospedeiras.

Palavras-chave: Anastrepha; Ceratitis capitata; Doryctobracon; Neosilba 


\section{Abstract}

New associations between Tephritidae and Lonchaeidae (Diptera) and their host plants in the eastern Amazon region. Larvae of Tephritidae and Lonchaeidae (Diptera) infest fruits of several plant species. The objective of this study was to identify the species of these families, their host plants and parasitoids in a mixed orchard in Belém, Pará in the eastern Amazon region. Samples of fruits of different plant species were collected every 30 days, from November 2017 to May 2018. A total of 109 fruit samples (2,355 fruits, $51.7 \mathrm{~kg})$ were collected from 21 plant species ( 8 native and 13 introduced) from 9 botanical families. There were infestations by frugivorous larvae in 63 samples (11 species of 6 botanical families). Specimens were obtained from six species of Tephritidae, three species of Lonchaeidae and five species of Braconidae parasitoids. Spondias mombin had the highest infestation index, showing the highest rate of parasitism. In this work, we found new associations between species of Tephritidae and Lonchaeidae and host plants.

Key words: Anastrepha; Ceratitis capitata; Doryctobracon; Neosilba

\section{Introdução}

As principais famílias de Diptera em que suas larvas utilizam a polpa dos frutos ou partes das plantas como substratos para seu desenvolvimento são Tephritidae e Lonchaeidae (ALMEIDA et al., 2016).

Em decorrência dos prejuízos diretos e indiretos que causam à produção de frutos, os insetos da família Tephritidae são considerados um dos maiores grupos com importância econômica mundial (ALUJA, 1994; ALUJA; MANGAN, 2008). Além disso, algumas espécies podem impossibilitar exportações em função das restrições quarentenárias impostas por países importadores que não apresentam uma determinada praga em seu território (MALAVASI, 2000; 2009).

Para as Américas, o gênero Anastrepha é considerado o de maior importância econômica (NORRBOM et al., 1999). No Brasil, seis espécies são particularmente importantes: Anastrepha striata Schiner, Anastrepha obliqua (Macquart), Anastrepha fraterculus (Wiedemann), Anastrepha grandis (Macquart), Anastrepha pseudoparallela (Loew) e Anastrepha zenildae Zucchi (URAMOTO; ZUCCHI, 2009). No entanto, a importância de qualquer espécie varia com a região, a disponibilidade hospedeira e a sua abundância. No país também ocorrem duas espécies exóticas introduzidas: Ceratitis capitata (Wiedemann), a mosca-do-mediterrâneo, distribuída em praticamente todo o território nacional (ZUCCHI, 2015), e Bactrocera carambolae Drew \& Hancock, a mosca-da-carambola, com distribuição restrita a localidades dos estados do
Amapá, Pará e Roraima, mantida sob controle oficial pelo Ministério da Agricultura, Pecuária e Abastecimento (BRASIL, 2018).

Nos últimos anos, especialmente no Brasil, dípteros da família Lonchaeidae têm sido reportados como invasores primários em espécies vegetais comerciais (ADAIME et al., 2017). Por exemplo, Neosilba zadolicha McAlpine \& Steyskal, Neosilba pendula (Bezzi), Neosilba glaberrima (Wiedemann) e Neosilba perezi (Romero \& Ruppel) são atualmente consideradas pragas, em razão dos danos causados em frutos, assim como em outras estruturas de diferentes espécies vegetais (LOURENÇÃO et al., 1996; UCHÔA, 2012; LEMOS et al., 2015). Recentemente, alguns estudos sobre Lonchaeidae foram realizados no Brasil, inclusive na Amazônia brasileira (LEMOS et al., 2015; PEREIRA; ADAIME, 2016). Entretanto, a escassez de informações sobre a taxonomia e bioecologia dessas espécies acabam dificultando o desenvolvimento de estratégias de manejo (STRIKIS et al., 2011).

Quanto aos inimigos naturais de larvas frugívoras, sua ação é um fator biótico que apresenta forte influência na regulação populacional de várias espécies. $\mathrm{Na}$ região Neotropical, os parasitoides pertencentes à família Braconidae estão entre os principais fatores de mortalidade de Anastrepha (CARVALHO et al., 2010). Na Amazônia brasileira, estão assinaladas oito espécies de Braconidae, sendo Doryctobracon areolatus (Szépligeti) e Opius bellus Gahan as mais abundantes e amplamente distribuídas, além de três espécies de Figitidae, com predomínio de Aganaspis 
pelleranoi (Brèthes) (ADAIME et al., 2018). No caso dos Lonchaeidae, oito espécies de parasitoides Eucoilinae (Figitidae) já foram registradas associadas a larvas de Neosilba no Brasil (UCHÔA, 2012). O parasitismo natural nas diferentes regiões produtoras de frutas no Brasil é variável, uma vez que é influenciado pela espécie vegetal hospedeira, pela mosca hospedeira, pelo ambiente e época de coleta (OVRUSKI et al., 2000).

A identificação das plantas hospedeiras de larvas frugívoras é fundamental para o estabelecimento de um programa de manejo adequado e eficiente para as espécies-praga (JESUS-BARROS et al., 2012). O tipo de amostragem mais adequado para determinar as relações mosca/hospedeiro/parasitoide de forma mais precisa é a coleta de frutos, que ainda determina os índices de infestação e parasitismo em cada hospedeiro (SILVA et al., 2011). Este trabalho teve o objetivo de identificar as espécies de Tephritidae e Lonchaeidae, suas plantas hospedeiras e parasitoides, em um pomar misto localizado em Belém, Pará, Amazônia Oriental.

\section{Material e Métodos}

\section{Caracterização da área de estudo}

O estudo foi realizado em um pomar misto agroecológico, com área aproximada de 4 ha, composto de aproximadamente 1.538 espécimes pertencentes a 50 espécies frutíferas, localizado na sede da Embrapa Amazônia Oriental (01²6'14,6”'S e 48²6’32,3”W, 10 $\mathrm{m}$ de altitude acima do nível do mar), em Belém, Pará. $\mathrm{O}$ clima da localidade é o equatorial $A f$ pela classificação de Köppen-Geiger, influenciado diretamente pela presença da floresta amazônica. A amplitude térmica é baixa, com temperatura média compensada anual de $26,5^{\circ} \mathrm{C}$, além de um índice pluviométrico anual superior a $3.000 \mathrm{~mm}$ (INMET, 2019).

\section{Procedimentos amostrais}

Foram realizadas coletas mensais de frutos carnosos de diversas espécies vegetais, de novembro de 2017 a maio de 2018, totalizando sete coletas, período em que há maior disponibilidade de plantas em frutificação na área de estudo.
Para quantificar o índice de infestação por larvas de dípteros e a percentagem de parasitismo, o método adotado foi o de amostras de frutos agrupados, detalhado por Silva et al. (2011). Na ocasião em que as plantas se apresentavam com boa carga de frutos, a amostra era composta por aproximadamente 50 frutos pequenos, 20 frutos médios ou 10 frutos grandes de uma mesma espécie vegetal (frutos em maturação ou já maduros, coletados diretamente da planta ou recém-caídos no solo). Em campo, os frutos coletados foram acondicionados em caixas térmicas com tampas vazadas e cobertas com tecido organza. Durante o transporte dos frutos do local de coleta até o Laboratório de Entomologia da Embrapa Amazônia Oriental, as caixas térmicas ficaram à sombra para evitar a mortalidade dos imaturos.

\section{Obtenção de pupários e de insetos adultos}

No laboratório, os frutos foram pesados e transferidos para bandejas de plástico, contendo uma fina camada de areia esterilizada umedecida. As bandejas foram cobertas com tecido tipo organza, preso por elásticos. A cada três dias o material contido nas bandejas foi examinado e os pupários retirados e transferidos para frascos de plástico transparentes ( $8 \mathrm{~cm}$ de diâmetro), contendo uma fina camada de vermiculita umedecida. Os frascos foram cobertos com organza, presa por tampa vazada. Em seguida, os frascos eram dispostos em câmaras climatizadas, tipo BOD, sob condições controladas de temperatura $\left(25 \pm 0,5^{\circ} \mathrm{C}\right)$, umidade relativa do ar $(70 \pm$ $10 \%)$ e fotofase $(12 \mathrm{~h})$, e observados diariamente para obtenção dos insetos adultos. Os insetos que emergiram (moscas e parasitoides) foram mortos e acondicionados em recipientes de vidro, contendo etanol $70 \%$, devidamente etiquetados, para posterior identificação.

\section{Identificação dos insetos}

Os exemplares de Anastrepha foram identificados com o auxílio da chave dicotômica ilustrada de Zucchi et al. (2011), baseando-se especialmente na forma do acúleo extrovertido da fêmea. A identificação de Ceratitis capitata foi realizada com base em Zucchi (2015). Para a identificação dos parasitoides (Braconidae) foram utilizados os trabalhos de Canal e Zucchi (2000), 
Marinho et al. (2011; 2017). Espécimes de Neosilba foram identificados pelo taxonomista Dr. Pedro Carlos Strikis, com base no exame da genitália do macho e/ou da quetotaxia da fêmea.

\section{Análise dos dados}

Foram calculados: 1) índice de infestação: número de pupários $/ \mathrm{Kg}=$ número de pupários obtidos/peso $(\mathrm{kg})$ frutos coletados; 2) percentual de parasitismo: (número de parasitoides emergidos/número de pupários) $\times 100$.

\section{Resultados}

Foram coletadas 109 amostras de frutos (2.355 frutos; $51,7 \mathrm{~kg}$ ) de 21 espécies vegetais (oito nativas e
13 introduzidas), pertencentes a nove famílias (Tabela 1). Por apresentarem significativa frutificação no período de amostragem, as espécies que propiciaram maior número de amostras foram: Averrhoa carambola $(27$ amostras), Garcinia acuminata (12), Myrciaria dubia (nove), Psidium araca (nove) e Garcinia brasiliensis (oito) (Tabela 1).

Houve infestação por larvas de dípteros em 63 amostras $(57,8 \%)$, pertencentes a 11 espécies vegetais de seis famílias (Tabela 2). Foram obtidos 3.086 pupários, dos quais emergiram espécimes de Tephritidae (seis espécies), Lonchaeidae (três espécies) e Hymenoptera (cinco espécies).

As espécies de Tephritidae obtidas foram: Anastrepha antunesi Lima, Anastrepha fraterculus

TABELA 1: Espécies vegetais amostradas para obtenção de Tephritidae e Lonchaeidae (Diptera) em um pomar misto na Amazônia Oriental. Belém, Pará, Brasil. Novembro de 2017 a maio de 2018.

\begin{tabular}{|c|c|c|c|c|c|c|c|c|c|c|}
\hline \multirow{2}{*}{ Nomes científicos } & \multirow{2}{*}{$\begin{array}{c}\text { Nomes } \\
\text { vernaculares }\end{array}$} & \multirow{2}{*}{ Famílias } & \multicolumn{7}{|c|}{ Amostras coletadas (n) } & \multirow{2}{*}{ Total } \\
\hline & & & Nov. & Dez. & Jan. & Fev. & Mar. & Abr. & Maio & \\
\hline Averrhoa carambola L. & Carambola & Oxalidaceae & 3 & 3 & 6 & 4 & 6 & 2 & 3 & 27 \\
\hline Byrsonima crassifolia (L.) Kunth & Murici & Malpighiaceae & 1 & 2 & & & & & & 3 \\
\hline Eugenia stipitata McVaugh & Araçá-boi & Myrtaceae & & 3 & & & & & & 3 \\
\hline Eugenia uniflora L. & Pitanga & Myrtaceae & 1 & & & 2 & & 1 & 2 & 6 \\
\hline Garcinia $\times$ mangostana $\mathrm{L}$. & Mangostão & Clusiaceae & & & & 1 & & & & 1 \\
\hline Garcinia acuminata Planch. \& Triana & Bacuri rugoso & Clusiaceae & 1 & 1 & 2 & 2 & 2 & 2 & 2 & 12 \\
\hline Garcinia brasiliensis Mart. & Bacuri liso & Clusiaceae & 2 & & 2 & 2 & & & 2 & 8 \\
\hline Garcinia humilis (Vahl) C.D.Adams & Achachairu & Clusiaceae & 1 & 1 & 2 & 2 & & & & 6 \\
\hline Garcinia macrophylla Mart. & Bacuripari & Clusiaceae & & 2 & & & 1 & & & 3 \\
\hline Malpighia emarginata DC. & Acerola & Malpighiaceae & 3 & & 1 & & & & & 4 \\
\hline Mammea americana $\mathrm{L}$. & Abricó & Clusiaceae & 1 & & & & & & & 1 \\
\hline Mangifera indica $\mathrm{L}$. & Manga cavalo & Anacardiaceae & & & 1 & & & & & 1 \\
\hline Manilkara zapota (L.) P.Royen & Sapotilha & Sapotaceae & & & & & & 1 & & 1 \\
\hline Morinda citrifolia $\mathrm{L}$. & Noni & Rubiaceae & & & 1 & & & & & 1 \\
\hline Myrciaria dubia (Kunth) McVaugh & Camu-camu & Myrtaceae & & 2 & 1 & 1 & 2 & 2 & 1 & 9 \\
\hline Nephelium lappaceum L. & Rambutã & Sapindaceae & 2 & 3 & 2 & & & & & 7 \\
\hline Psidium araca Raddi & Araçá-italiano & Myrtaceae & & & & 2 & 3 & 2 & 2 & 9 \\
\hline Spondias mombin L. & Taperebá & Anacardiaceae & & 2 & & & & & & 2 \\
\hline Syzygium jambos (L.) Alston & Jambo rosa & Myrtaceae & & & 1 & & 1 & & 1 & 3 \\
\hline Syzygium malaccense (L.) Merr. \& L.M Perry & Jambo comum & Myrtaceae & & & & & 1 & & & 1 \\
\hline Theobroma cacao L. & Cacau & Malvaceae & & & & 1 & & & & 1 \\
\hline Total & & & 15 & 19 & 19 & 17 & 16 & 10 & 13 & 109 \\
\hline
\end{tabular}


TABELA 2: Índices de infestação de frutos de várias espécies vegetais por larvas de Tephritidae e Lonchaeidae (Diptera) em um pomar misto na Amazônia Oriental. Belém, Pará, Brasil. Novembro de 2017 a maio de 2018.

\begin{tabular}{|c|c|c|c|c|c|c|c|c|}
\hline $\begin{array}{l}\text { FAMÍLIAS } \\
\text { Nomes científicos }\end{array}$ & $\begin{array}{l}\text { Origem } \\
\text { N/I }\end{array}$ & $\mathbf{I} / \mathbf{C}$ & $\begin{array}{l}\text { Frutos } \\
\text { (n) }\end{array}$ & $\begin{array}{c}\text { Massa } \\
(\mathbf{k g})\end{array}$ & $\begin{array}{l}\text { PP } \\
\text { (n) }\end{array}$ & $\begin{array}{c}\text { II } \\
(\mathrm{PP} / \mathrm{kg})\end{array}$ & $\begin{array}{c}\text { Emergência (Tephritidae; } \\
\text { Lonchaeidae; Hymenoptera) }\end{array}$ & $\begin{array}{c}P \\
(\%)\end{array}$ \\
\hline \multicolumn{9}{|l|}{ ANACARDIACEAE } \\
\hline Mangifera indica $\mathrm{L}$. & I & $0 / 1$ & 10 & 0,99 & & & & \\
\hline Spondias mombin $\mathrm{L}$. & $\mathrm{N}$ & $2 / 2$ & 100 & 1,20 & 560 & 466,7 & $\mathrm{Ao}(38), \operatorname{Af}(13), \mathrm{Aa}(1), 60 \overbrace{}^{\wedge} \mathrm{A} ; \mathrm{Nz}(1), 1$ $\mathrm{N} ; \mathrm{Da}(178), \mathrm{Ob}(117)$ & 52,7 \\
\hline \multicolumn{9}{|l|}{ CLUSIACEAE } \\
\hline Garcinia acuminata Planch. \& Triana & I & $4 / 12$ & 240 & 3,45 & 4 & 1,16 & $1 \oplus \mathrm{N}$ & \\
\hline Garcinia brasiliensis Mart. & $\mathrm{N}$ & $2 / 8$ & 160 & 2,22 & 4 & 1,80 & $\mathrm{~Np}(2), 1+\mathrm{N}$ & \\
\hline Garcinia humilis (Vahl) C.D.Adams & I & $0 / 6$ & 120 & 2,25 & & & & \\
\hline Garcinia macrophylla Mart. & $\mathrm{N}$ & $0 / 3$ & 50 & 1,83 & & & & \\
\hline Mammea americana $\mathrm{L}$. & I & $0 / 1$ & 10 & 1,64 & & & & \\
\hline Garcinia $\times$ mangostana $\mathrm{L}$ & I & $0 / 1$ & 10 & 0,71 & & & & \\
\hline \multicolumn{9}{|l|}{ MALPIGHIACEAE } \\
\hline Byrsonima crassifolia (L.) Kunth & $\mathrm{N}$ & $0 / 3$ & 150 & 0,39 & & & & \\
\hline Malpighia emarginata DC. & I & $4 / 4$ & 200 & 1,27 & 150 & 118,1 & 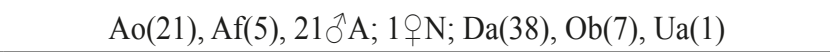 & 30,7 \\
\hline \multicolumn{9}{|l|}{ MALVACEAE } \\
\hline Theobroma cacao L. & I & $0 / 1$ & 05 & 1,34 & & & & \\
\hline \multicolumn{9}{|l|}{ MYRTACEAE } \\
\hline Eugenia stipitata McVaugh & $\mathrm{N}$ & $3 / 3$ & 30 & 4,65 & 214 & 46,0 & $\mathrm{Ao}(29), \operatorname{Af}(28), 59 \AA \mathrm{A} ; \mathrm{Da}(13), \mathrm{Ob}(1)$ & 6,5 \\
\hline Eugenia uniflora L. & $\mathrm{N}$ & $6 / 6$ & 300 & 1,92 & 307 & 159,9 & 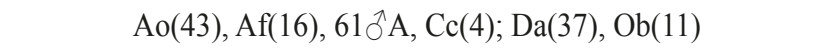 & 15,6 \\
\hline Myrciaria dubia (Kunth) McVaugh & $\mathrm{N}$ & $3 / 9$ & 450 & 3,02 & 15 & 4,97 & $\operatorname{Ao}(5), \operatorname{Af}(1), 10{ }^{\lambda} \mathrm{A}$ & \\
\hline Psidium araca Raddi & $\mathrm{N}$ & 9/9 & 90 & 4,61 & 443 & 96,1 & $\operatorname{As}(171), \operatorname{Ao}(2), 185{ }^{\wedge} \mathrm{A} ; \mathrm{Ng}(1), 1$ $\mathrm{N} ; \mathrm{Da}(16), \mathrm{Dd}(3), \mathrm{Ob}(1), \mathrm{Ua}(1)$ & 4,7 \\
\hline Syzygium jambos (L.) Alston & I & $2 / 3$ & 60 & 1,07 & 39 & 36,4 & $\operatorname{Ao}(6), \operatorname{Af}(3), 12 \widehat{A} ; \mathrm{Da}(6)$ & \\
\hline Syzygium malaccense (L.) Merr. \& L.M.Perry & $\mathrm{I}$ & $0 / 1$ & 10 & 1,12 & & & & \\
\hline \multicolumn{9}{|l|}{ OXALIDACEAE } \\
\hline Averrhoa carambola $\mathrm{L}$. & I & $27 / 27$ & 270 & 13,58 & 130 & 96,1 & $\mathrm{Ao}(254), \operatorname{Af}(51), 279{ }^{\Uparrow} \mathrm{A} ; \mathrm{Da}(165), \mathrm{Ob}(31), \mathrm{An}(7), \mathrm{Ua}(4)$ & 15,9 \\
\hline \multicolumn{9}{|l|}{ RUBIACEAE } \\
\hline Morinda citrifolia $\mathrm{L}$. & I & $0 / 1$ & 10 & 0,89 & & & & \\
\hline \multicolumn{9}{|l|}{ SAPINDACEAE } \\
\hline Nephelium lappaceum L. & I & $0 / 7$ & 70 & 2,41 & & & & \\
\hline \multicolumn{9}{|l|}{ SAPOTACEAE } \\
\hline Manilkara zapota (L.) P.Royen & I & $1 / 1$ & 10 & 1,11 & 45 & 40,5 & $\mathrm{Ap}(20), 14 \overbrace{}^{\lambda} \mathrm{A}$ & \\
\hline
\end{tabular}

N: nativa; I: introduzida; I: amostras infestadas; C: amostras coletadas; PP: pupários; II: infestação; P: percentual de parasitismo; Aa: Anastrepha antunesi; Af: Anastrepha fraterculus; Ao: Anastrepha obliqua; Ap: Anastrepha serpentina; As: Anastrepha striata; ${ }^{\top} \mathrm{A}$ : machos de Anastrepha; Cc: Ceratitis capitata; Ng: Neosilba glaberrima; Np: Neosilba pseudozadolicha; Nz: Neosilba zadolicha; ㅇN: fêmeas de Neosilba; An: Asobara anastrephae; Da: Doryctobracon areolatus; Dd: Doryctobracon adaimei; Ob: Opius bellus; Ua: Utetes anastrephae. 
(Wiedemann), Anastrepha obliqua (Macquart), Anastrepha serpentina (Wiedemann), Anastrepha striata Schiner e Ceratitis capitata (Wiedemann) (Tabela 2).

Anastrepha obliqua ocorreu em oito espécies vegetais hospedeiras (A. carambola, Eugenia stipitata, Eugenia uniflora, Malpighia emarginata, M. dubia, P. araca, Spondias mombin e Syzygium jambos), pertencentes a quatro famílias, predominando em Myrtaceae (Tabela 2). Desses hospedeiros, A. fraterculus não ocorreu apenas em $P$. araca. As demais espécies de Tephritidae ocorreram em apenas um hospedeiro cada: A. antunesi em S. mombin, A. striata em P. araca, A. serpentina em Manilkara zapota e C. capitata em E. uniflora.

As espécies de Lonchaeidae obtidas foram: Neosilba glaberrima (Wiedemann), Neosilba pseudozadolicha Strikis e Neosilba zadolicha McAlpine \& Steyskal (Tabela 2). Neosilba zadolicha ocorreu em S. mombin, $N$. pseudozadolicha em G. brasiliensis e N. glaberrima em $P$. araca.

Foram obtidas as seguintes espécies de Hymenoptera parasitoides: Asobara anastrephae (Muesebeck), Doryctobracon areolatus (Szépligeti), Doryctobracon adaimei Marinho \& Penteado-Dias, Opius bellus Gahan e Utetes anastrephae (Viereck) (Tabela 2).

O maior índice de infestação foi registrado em $S$. mombin (466,7 pupários/kg de fruto), predominando infestação por A. obliqua (Tabela 2). Nessa mesma espécie vegetal também foi registrado o maior percentual de parasitismo $(52,7 \%)$.

\section{Discussão}

Todas as espécies de Tephritidae obtidas neste trabalho já foram registradas no estado do Pará (ZUCCHI; MORAES, 2008; ADAIME et al., 2016), sendo quatro delas (A. fraterculus, A. obliqua, A. striata e $C$. capitata) consideradas de importância econômica para o Brasil (URAMOTO; ZUCCHI, 2009; ZUCCHI, 2015). Embora A. serpentina, neste trabalho obtida de frutos de $M$. zapota, não seja considerada importante economicamente para o país, no Pará, particularmente, tem significativa relevância pelo fato de infestar laranja, Citrus sinensis (L.), no polo de citricultura do município de Capitão Poço, como demonstrado por Lemos et al. (2011).

Anastrepha obliqua e A. fraterculus ocorreram em oito e sete hospedeiros, respectivamente (Tabela 2), confirmando que estão entre as espécies de moscasdas-frutas mais polífagas (ZUCCHI; MORAES, 2008). A ocorrência de $A$. obliqua e $A$. striata em $P$. araca constituem novas associações para o Brasil. Por sua vez, a ocorrência de $C$. capitata em $E$. uniflora constitui nova associação para a Amazônia brasileira. Até então, $C$. capitata foi registrada em outras sete espécies vegetais hospedeiras, sendo seis no Pará (CASTILHO et al., 2019).

Neosilba pseudozadolicha é reportada pela primeira vez no Pará. Até então, sua distribuição na Amazônia brasileira estava restrita ao estado do Amapá (PEREIRA; ADAIME, 2016). As associações Neosilba zadolicha em S. mombin, N. pseudozadolicha em G. brasiliensis e $N$. glaberrima em $P$. araca são inéditas para a Amazônia brasileira.

Entre os parasitoides, D. adaimei é reportado pela primeira vez no Pará, a partir de duas amostras de $P$. araca infestadas por $A$. striata. A espécie, recentemente descrita, estava assinalada apenas para os estados do Amapá e Tocantins (ZUCCHI; MORAES, 2008; MARINHO et al., 2017).

O índice de infestação registrado em $S$. mombin (466,7 pupários/kg) foi próximo ao maior valor já registrado na Amazônia brasileira (532,6 pupários/kg), em Amajari, Roraima (MARSARO JÚNIOR et al., 2010). O índice de parasitismo registrado em pupários de dípteros nessa espécie vegetal foi elevado $(52,7 \%)$, superior aos maiores índices já reportados na região, por exemplo, 46,2\% em Roraima (MARSARO JÚNIOR et al., 2011) e 46,9\% no Amapá (DEUS et al., 2013).

Este trabalho apresenta novas associações entre espécies de Tephritidae e Lonchaeidae e suas plantas hospedeiras na Amazônia Oriental, contribuindo para o conhecimento desses grupos biológicos. 


\section{Agradecimentos}

À Coordenação de Aperfeiçoamento de Pessoal de Nível Superior - CAPES, pela Bolsa de Doutorado concedida a Alison Pureza Castilho, e ao Conselho Nacional de Desenvolvimento Científico e Tecnológico - CNPq, pela Bolsa de Produtividade em Pesquisa concedida a Ricardo Adaime. Ao Dr. José Edmar Urano de Carvalho, pesquisador da Embrapa Amazônia Oriental, pelo apoio inestimável na realização deste trabalho. Ao Dr. Pedro Carlos Strikis, pela identificação dos Lonchaeidae.

\section{Referências}

ADAIME, R.; LIMA, A. L.; SOUSA, M. S. M. Controle biológico conservativo de moscas-das-frutas na Amazônia brasileira. Innovations Agronomiques, Paris, v. 64, p. 47-59, 2018.

ADAIME, R.; SOUSA, M. S. M.; JESUS-BARROS, C. R.; DEUS, E. G.; PEREIRA, J. F.; STRIKIS, P. C.; SOUZA-FILHO, M. F. Frugivorous flies (Diptera: Tephritidae, Lonchaeidae), their host plants, and associated parasitoids in the extreme north of Amapá State, Brazil. Florida Entomologist, Lutz, v. 100, n. 2, p. 316-324, 2017.

ADAIME, R.; SOUSA, M. S. M.; PEREIRA, J. F. Anastrepha species and their host in the Brazilian Amazon. 2016. Disponível em: <http://anastrepha.cpafap.embrapa.br>.

ALMEIDA, R. R.; CRUZ, K. R.; SOUSA, M. S. M.; COSTANETO, S. V.; JESUS-BARROS, C. R.; LIMA, A. L.; ADAIME, R. Frugivorous flies (Diptera: Tephritidae, Lonchaeidae) associated with fruit production on Ilha de Santana, Brazilian Amazon. Florida Entomologist, Lutz, v. 99, n. 3, p. 426-436, 2016.

ALUJA, M. Bionomics and management of Anastrepha. Annual Review of Entomology, Palo Alto, v. 39, n. 1, p. 155-178, 1994.

ALUJA, M.; MANGAN, R. L. Fruit fly (Diptera: Tephritidae) host status determination: critical conceptual, methodological, and regulatory considerations. Annual Review of Entomology, Palo Alto, v. 53, p. 473-502, 2008.

BRASIL. Instrução Normativa no 38, de 1 de outubro de 2018. 2018. Brasília: Diário Oficial [da] União, 2 out. 2018, Seção 1, 2018. p. 14.

CANAL, N. A.; ZUCCHI, R. A. Parasitoides - Braconidae. In: MALAVASI, A.; ZUCCHI, R. A. (Ed.). Moscas-das-frutas de importância econômica no Brasil: conhecimento básico e aplicado. Ribeirão Preto: Holos, 2000. p. 119-126.

CARVALHO, R. S.; SOARES FILHO, W. S.; RITZINGER, R. Umbu-cajá como repositório natural de parasitoide nativo de moscas-das-frutas. Pesquisa Agropecuária Brasileira, Brasília, v. 45, n. 10, p. 1222-1225, 2010.

CASTILHO,A. P.; BRANDÃO, C.A. C.; AYRES, A. R.; PEREIRA, J. F.; ADAIME, R. Distribuição geográfica e plantas hospedeiras de Ceratitis capitata (Wiedemann) (Diptera: Tephritidae) na
Amazônia brasileira. In: JASPER, M. (Org.). Coletânea Nacional Sobre Entomologia. Ponta Grossa: Atena Editora, 2019. [e-book]. DEUS, E. G.; PINHEIRO, L. S.; LIMA, C. R.; SOUSA, M. S. M.; GUIMARÃES, J. A.; STRIKIS, P. C.; ADAIME, R. Wild hosts of frugivorous dipterans (Tephritidae and Lonchaeidae) and associated parasitoids in the Brazilian Amazon. Florida Entomologist, Lutz, v. 96, n. 4, p. 1621-1625, 2013.

INMET - INSTITUTO NACIONAL DE METEOROLOGIA. Climatologia. 2019. Disponível em: <http://www.inmet.gov.br/ portal/index.php? $\mathrm{r}=$ clima/normaisClimatologicas $>$.

JESUS-BARROS, C. R.; ADAIME, R.; OLIVEIRA, M. N.; SILVA, W. R.; COSTA-NETO, S. V.; SOUZA-FILHO, M. F. Anastrepha (Diptera: Tephritidae) species, their hosts and parasitoids (Hymenoptera: Braconidae) in five municipalities of the State of Amapá, Brazil. Florida Entomologist, Lutz, v. 95, n. 3, p. $694-$ 705, 2012.

LEMOS, L. N.; ADAIME, R.; COSTA-NETO, S. V.; DEUS, E. G.; JESUS-BARROS, C. R.; STRIKIS, P. C. New findings on Lonchaeidae (Diptera: Tephritoidea) in the Brazilian Amazon. Florida Entomologist, Lutz, v. 98, n. 4, p. 1227-1237, 2015.

LEMOS, W. P.; SILVA, R. A.; ARAÚJO, S. C. A.; OLIVEIRA, E. L. A.; SILVA, W. R. First record of Anastrepha serpentina (Wiedemann) (Diptera: Tephritidae) in citrus in Brazil. Neotropical Entomology, Londrina, v. 40, n. 6, p. 706-707, 2011.

LOURENÇÃO, A. L.; LORENZI, J. O.; AMBROSANO, G. M. B. Comportamento de clones de mandioca em relação à infestação por Neosilba perezi (Romero \& Rupell) (Diptera: Lonchaeidae). Scientia Agricola, Piracicaba, v. 53, n. 2-3, p. 304-308, 1996.

MALAVASI, A. Áreas-livres ou de baixa prevalência. In: MALAVASI, A.; ZUCCHI, R. A. (Ed.). Moscas-das-frutas de importância econômica no Brasil: conhecimento básico e aplicado. Ribeirão Preto: Holos, 2000. p. 175-181.

MALAVASI, A. Biologia, ciclo de vida, relação com o hospedeiro, espécies importantes e biogeografia de tefritideos. In: MALAVASI, A.; VIRGÍNIO, J. (Ed.). Biologia, monitoramento e controle de moscas-das-frutas: V Curso Internacional de Capacitação em Moscas-das-frutas, 21 a 29 de outubro de 2009. Juazeiro: Biofábrica Moscamed Brasil, 2009. p. 1-15.

MARINHO, C. F.; CÔNSOLI, F. L.; PENTEADO-DIAS, A. M.; ZUCCHI, R. A. Description of two new species closely related to Doryctobracon areolatus (Szépligeti, 1911) (Hymenoptera, Braconidae), based on morphometric and molecular analyses. Zootaxa, Auckland, v. 4353, n. 3, p. 467-484, 2017.

MARINHO, C. F.; SILVA, R. A.; ZUCCHI, R. A. Chave de identificação de Braconidae (Alysiinae e Opiinae) parasitoides de larvas frugívoras na região Amazônica. In: SILVA, R. A.; LEMOS, W. P.; ZUCCHI, R. A. (Ed.). Moscas-das-frutas na Amazônia brasileira: diversidade, hospedeiros e inimigos naturais. Macapá: Embrapa Amapá, 2011. p. 91-101.

MARSARO JÚNIOR, A. L.; ADAIME, R.; RONCHI-TELES, B.; LIMA, C. R.; PEREIRA, P. R. V. S. Anastrepha species (Diptera: Tephritidae), their hosts and parasitoids in the extreme north of Brazil. Biota Neotropica, Campinas, v. 11, p. 117-124, 2011.

MARSARO JÚNIOR, A. L.; SILVA, R. A.; SILVA, W. R.; LIMA, C. R.; FLORES, A. S.; RONCHI-TELES, B. New records of Anastrepha (Diptera: Tephritidae), its hosts and parasitoids in the 
Serra do Tepequém, Roraima state, Brazil. Revista de Agricultura, Piracicaba, v. 85, n. 1, p. 15-20, 2010.

NORRBOM, A. L.; ZUCCHI, R. A.; HERNANDEZ-ORTIZ, V. Phylogeny of the genera Anastrepha and Toxotrypana (Trypetinae: Toxotripanini) based on morphology. In: ALUJA, M.; NORRBOM, A. L. (Ed.). Fruit flies (Tephritidae): phylogeny and evolution of behavior. Boca Raton: CRC Press, 1999. p. 299-342.

OVRUSKI, S. M.; ALUJA, M.; SIVINSKI, J.; WHARTON, R. A. Hymenopteran parasitoids on fruit-infesting Tephritidae (Diptera) in Latin America and the Southern United States: diversity, distribution, taxonomic status and their use in fruit fly biological control. Integrated Pest Management Reviews, Annapolis, v. 5, n. 2, p. 81-107, 2000.

PEREIRA, J. F.; ADAIME, R. Lonchaeidae from Brazilian Amazon. 2016. Disponível em: <http://lonchaeidae.cpafap. embrapa.br>.

SILVA, R. A.; DEUS, E. G.; RAGA, A.; PEREIRA, J. D. B.; SOUZA-FILHO, M. F.; COSTA NETO, S. V. Monitoramento de moscas-das-frutas na Amazônia: amostragem de frutos e uso de armadilhas. In: SILVA, R. A.; LEMOS, W. P.; ZUCCHI, R. A. (Ed.). Moscas-das-frutas na Amazônia brasileira: diversidade, hospedeiros e inimigos naturais. Macapá: Embrapa Amapá, 2011. p. 33-50.

STRIKIS, P. C.; DEUS, E. G.; SILVA, R. A.; PEREIRA, J. D. B.; JESUS, C. R.; MARSARO JÚNIOR, A. L. Conhecimento sobre Lonchaeidae na Amazônia brasileira. In: SILVA, R. A.; LEMOS, W. P.; ZUCCHI, R. A. (Ed.). Moscas-das-frutas na Amazônia brasileira: diversidade, hospedeiros e inimigos naturais. Macapá: Embrapa Amapá, 2011. p. 205-216.
UCHÔA, M. A. Fruit flies (Diptera: Tephritoidea): biology, host plants, natural enemies, and the implications to their natural control. In: LARRAMENDY, M. L.; SOLONESKI, S. (Ed.). Integrated pest management and pest control: current and future tactics. Rijeka: InTech, 2012. p. 271-300.

URAMOTO, K.; ZUCCHI, R. A. Taxonomia de espécies de Anastrepha (Diptera, Tephritidae). In: MALAVASI, A.; VIRGÍNIO, J. (Ed.). Biologia, monitoramento e controle de moscas-dasfrutas: V Curso Internacional de Capacitação em Moscas-dasfrutas, 21 a 29 de outubro de 2009. Juazeiro: Biofábrica Moscamed Brasil, 2009. p. 7-11.

ZUCCHI, R. A. Mosca-do-mediterrâneo, Ceratitis capitata (Wiedemann). In: VILELA, E. F.; ZUCCHI, R. A. (Ed.). Pragas introduzidas no Brasil: insetos e ácaros. Piracicaba: FEALQ, 2015. p. 153-172.

ZUCCHI, R. A.; MORAES, R. C. B. Fruit flies in Brazil Anastrepha species their host plants and parasitoids. 2008. Disponível em: <www.lea.esalq.usp.br/anastrepha/>.

ZUCCHI, R. A.; URAMOTO, K.; SOUZA-FILHO, M. F. Chave ilustrada para as espécies de Anastrepha da região Amazônica. In: SILVA, R. A.; LEMOS, W. P.; ZUCCHI, R. A. (Ed.). Moscasdas-frutas na Amazônia brasileira: diversidade, hospedeiros e inimigos naturais. Macapá: Embrapa Amapá, 2011. p. 71-90. 